\title{
Cost Analysis of Java Bytecode
}

\author{
E. Albert ${ }^{1}$, P. Arenas ${ }^{1}$, S. Genaim ${ }^{2}$, G. Puebla ${ }^{2}$, and D. Zanardini ${ }^{2}$ \\ ${ }^{1}$ DSIC, Complutense University of Madrid, E-28040 Madrid, Spain \\ ${ }^{2}$ CLIP, Technical University of Madrid, E-28660 Boadilla del Monte, Madrid, Spain
}

\begin{abstract}
Cost analysis of Java bytecode is complicated by its unstructured control flow, the use of an operand stack and its object-oriented programming features (like dynamic dispatching). This paper addresses these problems and develops a generic framework for the automatic cost analysis of sequential Java bytecode. Our method generates cost relations which define at compile-time the cost of programs as a function of their input data size. To the best of our knowledge, this is the first approach to the automatic cost analysis of Java bytecode.
\end{abstract}

\section{Introduction}

Cost analysis has been intensively studied in the context of declarative (see, e.g., [17 16 18 125] for functional programming and [1011] for logic programming) and high-level imperative programming languages (mainly focused on the estimation of worst case execution times and the design of cost models [23]). Traditionally, cost analysis has been formulated at the source level. However, there are situations where we do not have access to the source code, but only to compiled code. An example of this is mobile code, where the code consumer receives code to be executed. In this context, Java bytecode [13] is widely used, mainly due to its security features and the fact that it is platform-independent. Automatic cost analysis has interesting applications in this context. For instance, the receiver of the code may want to infer cost information in order to decide whether to reject code which has too large cost requirements in terms of computing resources (in time and/or space), and to accept code which meets the established requirements 8123. In fact, this is the main motivation for the Mobile Resource Guarantees (MRG) research project [3], which establishes a Proof-Carrying Code [15] framework for guaranteeing resource consumption. Furthermore, the Mobility, Ubiquity and Security (MOBIUS) research project [4, also considers resource consumption as one of the central properties of interest for proof-carrying code. Also, in parallel systems, knowledge about the cost of different procedures can be used in order to guide the partitioning, allocation and scheduling of parallel processes.

The aim of this work is to develop an automatic approach to the cost analysis of Java bytecode which statically generates cost relations. These relations define the cost of a program as a function of its input data size. This approach was proposed by Debray and Lin [10] for logic programs, and by Rabhi and Manson [16] for functional programs. In these approaches, cost functions are expressed by means of recurrence equations generated by abstracting the recursive

R. De Nicola (Ed.): ESOP 2007, LNCS 4421, pp. 157-172, 2007.

(C) Springer-Verlag Berlin Heidelberg 2007 
structure of the program and by inferring size relations between arguments. A low-level object-oriented language such as Java bytecode introduces novel challenges, mainly due to: 1) its unstructured control flow, e.g., the use of goto statements rather than recursive structures; 2) its object-oriented features, like virtual method invocation, which may influence the cost; and 3) its stack-based model, in which stack cells store intermediate values. This paper addresses these difficulties and develops a generic framework for the automatic cost analysis of Java bytecode programs. The process takes as input the bytecode corresponding to a method and yields a cost relation after performing these steps:

1. The input bytecode is first transformed into a control flow graph (CFG). This allows making the unstructured control flow of the bytecode explicit (challenge 1 above). Advanced features like virtual invocation and exceptions are simply dealt as additional nodes in the graph (challenge 2).

2. The CFG is then represented as a set of rules by using an intermediate recursive representation in which we flatten the local stack by converting its contents into a series of additional local variables (challenge 3) 1

3. In the third step, we infer size relations among the input variables for all calls in the rules by means of static analysis. These size relations are constraints on the possible values of variables (for integers) and constraints on the length of the longest reachable path (for references).

4. The fourth phase provides, for each rule of the recursive representation, a safe approximation of the set of input arguments which are "relevant" to the cost. This is performed using a simple static analysis.

5. From the recursive representation, its relevant arguments, and the size relations, the fifth step automatically yields as output the cost relation which expresses the cost of the method as a function of its input arguments.

We point out that computed cost relations, in many cases, can be simplified to the point of deriving statically an upper and lower threshold cost for the input size arguments and/or obtaining a closed form solution. Such simplifications have been well-studied in the field of algorithmic complexity (see e.g. 22]).

\section{The Java Bytecode Language}

Java bytecode [13] is a low-level object-oriented programming language with unstructured control and an operand stack to hold intermediate computational results. Moreover, objects are stored in dynamic memory (the heap). A Java bytecode program consists of a set of class files, one for each class or interface. A class file contains information about its name $c \in$ Class_Name, the class it extends, the interfaces it implements, and the fields and methods it defines. In particular, for each method, the class file contains: a method signature $m \in$ Meth_Sig which consists of its name name $(m) \in$ Meth_Name and its

\footnotetext{
${ }^{1}$ Note that this is possible since in every valid bytecode program the height of the
} local stack at each program point is fixed and therefore can be computed statically. 
type type $(m)=\tau_{1}, \ldots, \tau_{n} \rightarrow \tau \in$ Meth_Type where $\tau, \tau_{i} \in$ Type; its bytecode $b c_{m}=\left\langle p c_{0}: b_{0}, \ldots, p c_{n_{m}}: b_{n_{m}}\right\rangle$, where each $b_{i}$ is a bytecode instruction and $p c_{i}$ is its address; and the method's exceptions table. When it is clear from the context, we omit bytecode addresses and refer to a method signature as method.

In this work we consider a subset of the JVM [13] language which is able to handle operations on integers, object creation and manipulation (by accessing fields and calling methods) and exceptions (either generated by abnormal execution or explicitly thrown by the program). We omit interfaces, static fields and methods and primitive types different from integers. Methods are assumed to return an integer value. Thus, our bytecode instruction set (bcInst) is:

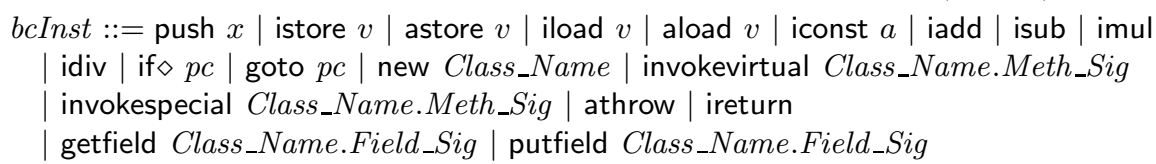

where $\diamond$ is a comparison operator (ne,le,_icmpgt, etc.), $v$ a local variable, $a$ an integer, $p c$ an instruction address, and $x$ an integer or the special value NULL.

\section{From Bytecode to Control Flow Graphs}

This section describes the generation of a control flow graph (CFG) from the bytecode of a method. This will allow transforming the unstructured control flow of bytecode into recursion. The technique we use follows well-established ideas in compilers 1, already applied in Java bytecode analysis 19.

Given a method $m$, we denote by $G_{m}$ its CFG, which is a directed graph whose nodes are referred to as blocks. Each block Block $k_{i d}$ is a tuple of the form $\langle i d, G, B, D\rangle$ where: $i d$ is the block's unique identifier; $G$ is the guard of the block which indicates under which conditions the block is executed; $B$ is a sequence of contiguous bytecode instructions which are guaranteed to be executed unconditionally (i.e., if $G$ succeeds then all instructions in $B$ are executed before control moves to another block); and $D$ is the adjacency list for Block $k_{i d}$, i.e., $D$ contains the identifiers of all blocks which are possible successors of Block $i d$, i.e., $i d^{\prime} \in D$ iff there is an arc from Block $k_{i d}$ to $B l o c k_{i d^{\prime}}$. Guards originate from bytecodes where the execution might take different paths depending on the runtime values. This is the case of bytecodes for conditional jumps, method invocation, and exceptions manipulation. In the CFG this will be expressed by branching from the corresponding block. The successive blocks will have mutually exclusive guards since only one of them will be executed. Guards take the form guard(cond), where cond is a Boolean condition on the local variables and stack elements of the method. It is important to point out that guards in the successive blocks will not be taken into account when computing the cost of a program.

A large part of the bytecode instruction set has only one successor. However, there are three types of branching statements:

Conditional jumps: of the form " $p c_{i}$ : if $\diamond p c_{j}$ ". Depending on the truth value of the condition, the execution can jump to $p c_{j}$ or continue, as usual, with $p c_{i+1}$. 
The graph describes this behavior by means of two arcs from the block containing the instruction of $p c_{i}$ to those starting respectively with instructions of $p c_{j}$ and $p c_{i+1}$. Each one of these new blocks begins by a guard expressing the condition under which such block is to be executed.

Dynamic dispatch: of the form " $p c_{i}$ : invokevirtual $c . m$ ". The type of the object $o$ whose method is being invoked is not known statically (it could be $c$ or any subclass of $c$ ); therefore, we cannot determine statically which method is going to be invoked. Hence, we need to make all possible choices explicit in the graph. We deal with dynamic dispatching by using the function resolve_virtual $(c, m)$, which returns the set ResolvedMethods of pairs $\left\langle d,\left\{c_{1}, \ldots, c_{k}\right\}\right\rangle$, where $d$ is a class that defines a method with signature $m$ and each $c_{i}$ is either $c$ or a subclass of $c$ which inherits that specific method from $d$. For each $\left\langle d,\left\{c_{1}, \ldots, c_{k}\right\}\right\rangle \in$ ResolvedMethods, a new block $B l o c k_{d}^{p c_{i}}$ is generated with a unique instruction invoke $(d: m)$ which stands for the non-virtual invocation of the method $m$ that is defined in the class $d$. In addition, the block has a guard of the form instanceof $\left(o,\left\{c_{1}, \ldots, c_{k}\right\}\right)$ (o is a stack element) to indicate that the block is applicable only when $o$ is an instance of one of the classes $c_{1}, \ldots, c_{k}$. An arc from the block containing $p c_{i}$ to $B l o c k_{d}^{p c_{i}}$ is added, together with an arc from Block ${ }_{d}^{p c_{i}}$ to the block containing the next instruction at $p c_{i+1}$ (which describes the rest of the execution after invoking $m$ ). Note that the invokevirtual is no longer needed in the CFG since it was split into several invoke instructions which cover all the possible runtime scenarios. Yet, in order to take into account the cost of dynamic dispatching, we replace the invokevirtual by a corresponding call to resolve_virtual. Fields are treated in a similar way.

Exceptions: As regards the structure of the CFG, exceptions are not dealt with in a special way. Instead, the possibility of an exception being raised while executing a bytecode statement $b$ is simply treated as an additional branching after $b$. Let $B l o c k_{b}$ be the block ending with $b$; arcs exiting from Block $k_{b}$ are those originated by its normal behavior control flow, together with those reaching the sub-graphs which correspond to exception handlers.

Describing dynamic dispatching and exceptions as additional blocks simplifies program analysis. After building the CFG, we do not need to distinguish how and why blocks were generated. Instead, all blocks can be dealt with uniformly.

Example 1 (running example). The execution of the method add $(\mathrm{n}, \mathrm{o})$ shown in Fig. 1 computes: $\Sigma_{i=0}^{n} i$ if o is an instance of $A ; \Sigma_{i=0}^{\lfloor n / 2\rfloor} 2 i$ if o is an instance of $B$; and $\Sigma_{i=0}^{\lfloor n / 3\rfloor} 3 i$ if $\mathrm{o}$ is an instance of $C$. The CFG of the method add is depicted at the bottom of the figure. The fact that the successor of 6: if_icmpgt 16 can be either the instruction at address 7 or 16 is expressed by means of two arcs from Block , one to Block $_{2}$ and another one to Block ${ }_{3}$, and by adding the guards icmpgt and icmple to Block $_{2}$ and Block $_{3}$, respectively. The invocation 13: invokevirtual A.incr: (I)I is split into 3 possible runtime scenarios de-

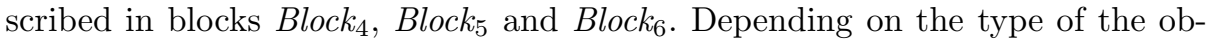
ject $\circ$ (the second stack element from top, denoted $s(\operatorname{top}(1))$ in the guards), 


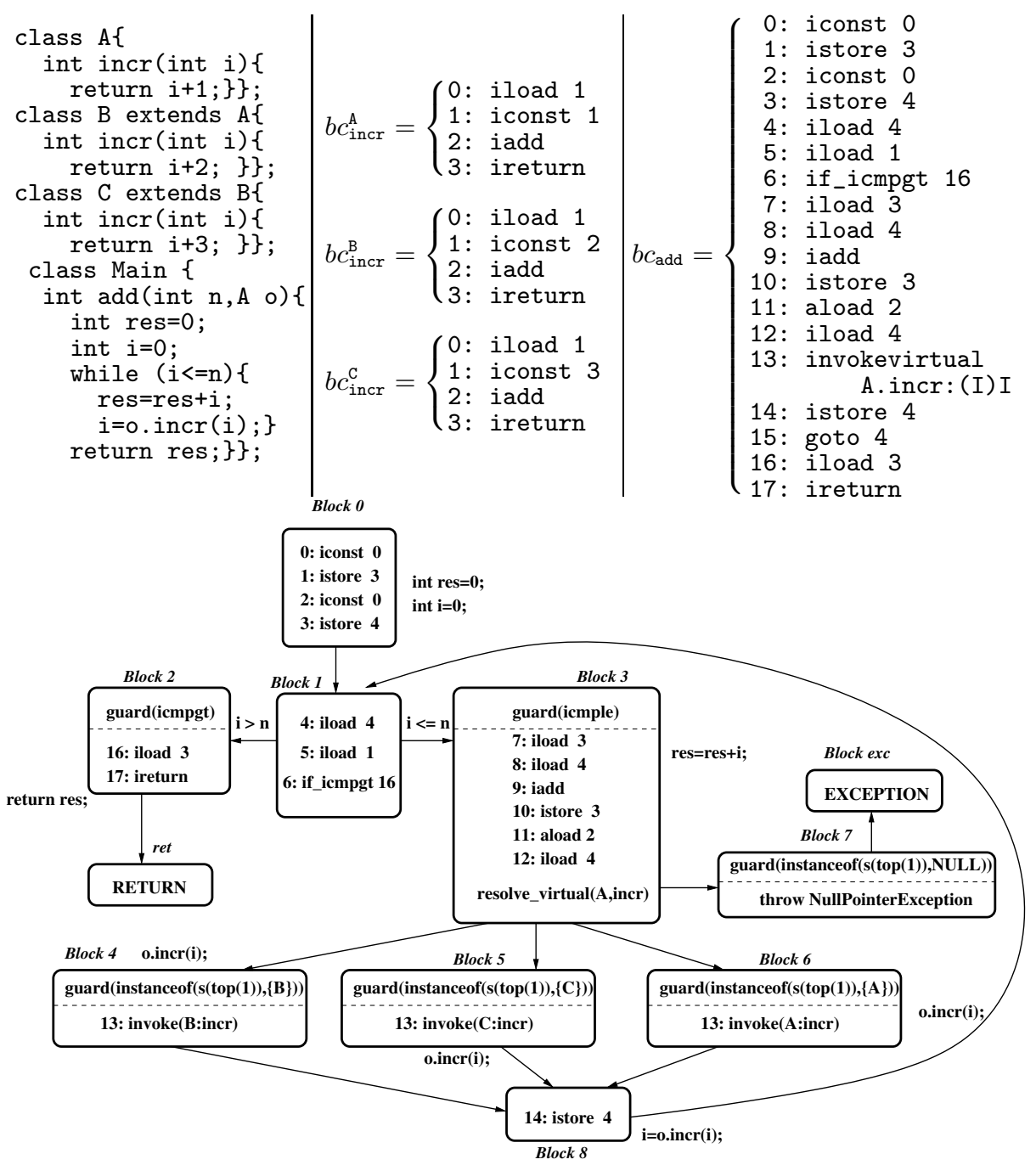

Fig. 1. The running example in source code, bytecode, and control flow graph

only one of these blocks will be executed and hence one of the definitions for incr will be invoked. Note that the invokevirtual bytecode is replaced by resolve_virtual. The exception behavior when o is a NULL object is described in blocks Block $_{7}$ and Block $k_{\text {exc }}$.

\section{Recursive Representation with Flattened Stack}

In this section, we present a method for obtaining a representation of the code of a method where 1) iteration is transformed into recursion and 2) the operand 
stack is flattened in the sense that its contents are represented as a series of local variables. The latter is possible because in valid bytecode the maximum stack height $t$ can always be statically decided. For the sake of simplicity, exceptions possibly occurring in a method will be ignored. Handling them introduces more branching in the CFG and also requires additional arguments in the recursive representation. This could influence the performance of the cost analysis.

Let $m$ be a method defined in class $c$, with local variables $\overline{l_{k}}=l_{0}, \ldots, l_{k}$; of them, $l_{0}$ contains a reference to the this object, $l_{1}, \ldots, l_{n}$ are the $n$ input arguments to the method, and $l_{n+1}, \ldots, l_{k}$ correspond to the $k-n$ local variables declared in $m$. In addition to these arguments, we add the variables $\overline{s_{t}}=s_{0}, \ldots, s_{t-1}$, which correspond to the stack elements, with $s_{0}$ and $s_{t-1}$ being the bottom-most and top-most positions respectively. Moreover, let $h_{i d}$ be the height of the stack at the entry of $B l o c k_{i d}$, and $\left.\overline{s_{t}}\right|_{h_{i d}}$ be the restriction of $\overline{s_{t}}$ to the corresponding stack variables. The recursive representation of $m$ is defined as a set of rules head $\leftarrow$ body obtained from its control flow graph $G_{m}$ as follows:

(1) the method entry rule is $\mathrm{c}: \mathrm{m}\left(\overline{\overline{1}_{\mathrm{n}}}\right.$, ret $) \leftarrow \mathrm{c}: \mathrm{m}^{0}\left(\overline{\overline{1}_{\mathrm{k}}}\right.$, ret $)$, where ret is a variable for storing the return value,

(2) for each $B l o c k_{i d}=\left\langle i d, G, \bar{B}_{p},\left\{i d_{1}, \ldots, i d_{j}\right\}\right\rangle \in G_{m}$, there is a rule:

$$
\mathrm{c}: \mathrm{m}^{i d}\left(\overline{\mathrm{I}_{\mathrm{k}}},\left.\overline{\mathrm{s}_{\mathrm{t}}}\right|_{\mathrm{h}_{\mathrm{id}}}, \text { ret }\right) \leftarrow \mathrm{G}^{\prime}, \overline{\mathrm{B}}_{\mathrm{p}}^{\prime}\left(\mathrm{call}_{\mathrm{id}_{1}} ; \ldots ; \operatorname{call}_{\mathrm{id}_{\mathrm{j}}}\right)
$$

where $\left\{\mathrm{G}^{\prime}\right\} \cup \overline{\mathrm{B}}_{\mathrm{p}}^{\prime}$ is obtained from $\{\mathrm{G}\} \cup \overline{\mathrm{B}}_{\mathrm{p}}$, and $\mathrm{call}_{\mathrm{id}_{1}} ; \ldots ; \mathrm{call}_{\mathrm{id}}$ are possible calls to blocks (";" means disjunction), as explained below.

Each $b_{i} \in\{\mathrm{G}\} \cup \overline{\mathrm{B}}_{\mathrm{p}}$ is translated into $b_{i}^{\prime}$ by explicitly adding the variables (local variables or stack variables) used by $b_{i}$ as arguments. For example, iadd is translated to iadd $\left(s_{j-1}, s_{j}, s_{j-1}^{\prime}\right)$, where $j$ is the index of the top of the stack just before executing iadd. Here, we refer to the $j-1^{\text {th }}$ stack variable twice by different names: $s_{j-1}$ refers to the input value and $s_{j-1}^{\prime}$ refers to the output value. The use of new names for output variables, in the spirit of Static Single Assignment ( $S S A$ ) (see [9] and its references), is crucial in order to obtain simple, yet efficient, denotational program analyses. In Fig. 2 we give the translation function for selected bytecodes; among them, the one for iadd works as follows. Function translate takes as input the name of the current method $m$, the program counter $p c$ of the bytecode, the bytecode (in this case iadd), the current local variable names $\overline{\mathrm{I}_{\mathrm{k}}}$, and the current stack variable names $\overline{\mathrm{s}_{\mathrm{t}}}$. In line 1 , we retrieve the index of the top stack element before executing the current bytecode. In line 2 , we generate new stack variable names $\overline{\mathbf{s}^{\prime}}{ }_{\mathrm{t}}$ by renaming the output variable of iadd in $\overline{\mathrm{s}}_{\mathrm{t}}$. As notation, given a sequence $\bar{a}_{n}$ of elements, $\bar{a}_{n}[i \mapsto b]$ denotes the replacement in $\bar{a}_{n}$ of the element $a_{i}$ by $b$. In line 3 , we return (ret $\left.\left\langle_{-}\right\rangle\right)$the translated bytecode together with the new stack variable names. Assume that $\mathrm{G}=p c_{0}: b_{0}$ and $\overline{\mathrm{B}}_{\mathrm{p}}=\left\langle p c_{1}: b_{1}, \ldots, p c_{\mathrm{p}}: b_{\mathrm{p}}\right\rangle$. The translation of all bytecodes is done iteratively as follows:

$$
\text { for } \mathrm{i}=0 \text { to } \mathrm{p}\left\{\left\langle b_{\mathrm{i}}^{\prime}, \overline{\mathrm{I}}_{\mathrm{k}}^{\mathrm{i}+1}, \overline{\mathrm{s}}_{\mathrm{t}}^{\mathrm{i}+1}\right\rangle=\operatorname{translate}\left(\mathrm{m}, p c_{\mathrm{i}}, b_{\mathrm{i}}, \overline{\mathrm{I}}_{\mathrm{k}}^{\mathrm{i}}, \overline{\mathrm{s}}_{\mathrm{t}}^{\mathrm{i}}\right)\right\}
$$

We start from an initial set of local and stack variables, $\overline{\mathrm{l}}_{\mathrm{k}}^{0}=\overline{\mathrm{l}}_{\mathrm{k}}$ and $\overline{\mathrm{s}}_{\mathrm{t}}^{0}=\overline{\mathrm{s}}_{\mathrm{t}}$; in each step, translate takes as input the local and stack variable names which 


\begin{tabular}{|c|c|}
\hline 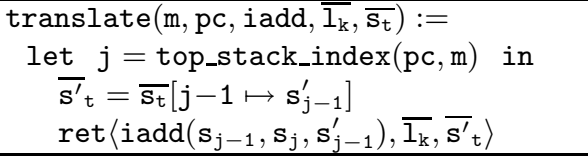 & $\begin{array}{l}\text { translate }\left(\mathrm{m}, \mathrm{pc}, \text { iload }(\mathrm{v}), \overline{\mathrm{I}}_{\mathrm{k}}, \overline{\mathbf{s}}_{\mathrm{t}}\right):= \\
\text { let } \mathrm{j}=\text { top_stack_index }(\mathrm{pc}, \mathrm{m}) \text { in } \\
{\overline{\mathbf{s}^{\prime}}}_{\mathrm{t}}=\overline{\mathbf{s}}_{\mathrm{t}}\left[\mathrm{j}+1 \mapsto \mathbf{s}_{\mathrm{j}+1}^{\prime}\right] \\
\quad \text { ret }\left\langle\operatorname{iload}\left(\mathrm{I}_{\mathrm{v}}, \mathbf{s}_{\mathrm{j}+1}^{\prime}\right), \overline{\mathrm{I}}_{\mathrm{k}},{\overline{\mathbf{s}^{\prime}}}_{\mathrm{t}}\right\rangle\end{array}$ \\
\hline 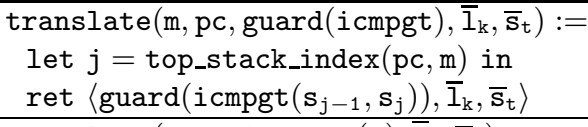 & $\begin{array}{l}\text { translate }\left(\mathrm{m}, \mathrm{pc}, \text { invoke }\left(\mathrm{b}: \mathrm{m}^{\prime}\right), \overline{\mathrm{I}}_{\mathrm{k}}, \overline{\mathrm{s}}_{\mathrm{t}}\right):= \\
\text { let } \mathrm{j}=\text { top_stack_index }(\mathrm{pc}, \mathrm{m}), \\
\mathrm{n}=\text { number_of_arguments }\left(\mathrm{b}, \mathrm{m}^{\prime}\right) \text { in }\end{array}$ \\
\hline $\begin{array}{l}\operatorname{translate}\left(\mathrm{m}, \mathrm{pc}, \text { ireturn }(\mathrm{v}), \overline{\mathrm{I}}_{\mathrm{k}}, \overline{\mathrm{s}}_{\mathrm{t}}\right):= \\
\operatorname{ret}\left\langle\operatorname{ireturn}\left(\mathrm{s}_{0}, \text { ret }\right), \overline{\mathrm{I}}_{\mathrm{k}}, \overline{\mathrm{s}}_{\mathrm{t}}\right\rangle\end{array}$ & $\begin{array}{l}{\overline{s^{\prime}}}_{t}=\bar{s}_{t}\left[j-n \mapsto s_{j-n}^{\prime}\right] \\
\text { ret }\left\langle b: m^{\prime}\left(s_{j-n}, \ldots, s_{j}, s_{j-}^{\prime}\right.\right.\end{array}$ \\
\hline
\end{tabular}

Fig. 2. Translation of selected bytecode instructions

were generated by translating the previous bytecode. At the end of this loop, we can define each $\mathrm{call}_{\mathrm{id}_{\mathrm{i}}}, 1 \leq i \leq j$, as $\mathrm{c}: \mathrm{m}^{\mathrm{id}}\left(\overline{\mathrm{l}}_{\mathrm{k}}^{\mathrm{p}+1},\left.\overline{\mathrm{s}}_{\mathrm{t}}^{\mathrm{p}+1}\right|_{\mathrm{h}_{\mathrm{id}_{\mathrm{i}}}}\right.$, ret $)$, meaning that we call the next block with the last local and (restricted) stack variable names.

Example 2. Consider the CFG in Fig. 1. The translation of Block $k_{3}$ and Block 4 works as shown below. For clarity, in the block identifiers we have not included the class name for the add method. Also, we ignore the exception branch from Block $_{3}$ to Block $_{7}$.

\begin{tabular}{|c|c|}
\hline 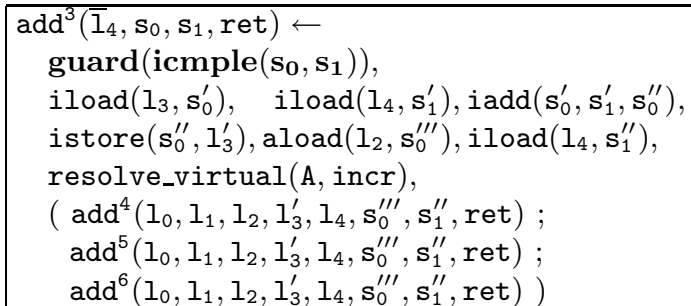 & $\begin{array}{l}\operatorname{add}^{4}\left(\overline{\mathbf{I}}_{4}, \mathbf{s}_{0}, \mathbf{s}_{1}, \text { ret }\right) \leftarrow \\
\quad \operatorname{guard}\left(\text { instanceof }\left(\mathbf{s}_{\mathbf{0}},\{\mathbf{B}\}\right)\right), \\
\quad \mathrm{B}: \operatorname{incr}\left(\mathbf{s}_{0}, \mathbf{s}_{1}, \mathbf{s}_{0}^{\prime}\right), \\
\quad \operatorname{add}^{8}\left(\overline{\mathbf{I}}_{4}, \mathbf{s}_{0}^{\prime}, \text { ret }\right) .\end{array}$ \\
\hline
\end{tabular}

In the $\operatorname{add}^{3}$ rule, dynamic dispatch is represented as a disjunction of calls to $\operatorname{add}^{4}, \operatorname{add}^{5}$ or $\operatorname{add}^{6}$. Thus, in the rule for $\operatorname{add}^{4}$, we find a call to (the translation of) incr from class B which corresponds to the translation of invoke(B:incr); arguments passed to incr are the two top-most stack elements; the return value (the last argument) goes also to the stack. Note the change in the superscript when a variable is updated.

Several optimizations are applied to the above translation. An important one is to replace (redundant) stack variables corresponding to intermediate states by local variables whenever possible. This can be done by tracking dependencies between variables, which stem from instructions like iload and istore. The fact that the program is in SSA form makes this transformation relatively straightforward. However, note that, in order to eliminate stack variables from the head of a block, we need to consider all calling patterns to the block. 
Example 3. After eliminating redundant variables, the optimized version of rules 3 and 4 from Ex. 2 is as follows:

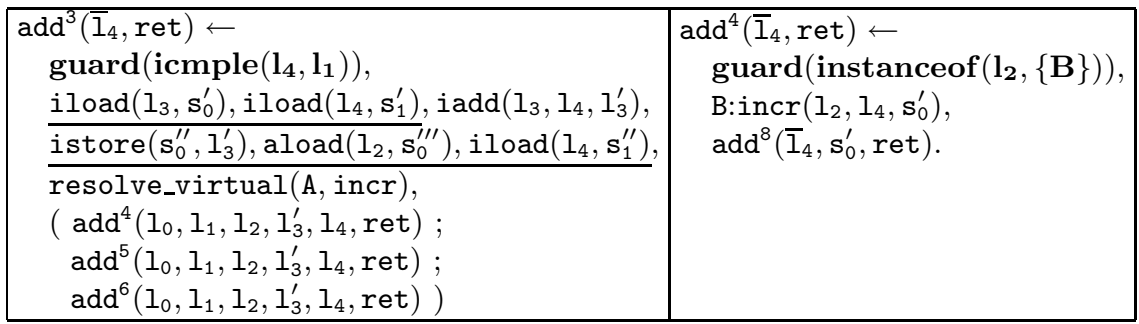

The underlined instructions have been used to discover equivalences among stack elements and local variables. For example, all the arguments of iadd have been replaced by local variables. However, eliminating stack variables is not always possible. This is the case of $\mathbf{s}_{0}^{\prime}$ in the rule add ${ }^{4}$, as it corresponds to the return value of B:incr. After these optimizations, the underlined instructions become redundant and could be removed. However, we do not remove them in order to take their cost into account in the next sections.

\section{Size Relations for Cost Analysis}

Obtaining size-relations between the states at different program points is indispensable for setting up cost relations. In particular, they are essential for defining the cost of one block in terms of the cost of its successors. In general, various measures can be used to determine the size of an input. For instance, in symbolic languages (see, e.g., [10]), term-depth, list-length, etc. are used as term sizes. In Java bytecode, we consider two cases: for integer variables, size-relations are constraints on the possible values of variables; for reference variables, they are constraints on the length of the longest reachable paths.

Example 4. Consider the two loops below, written in Java for simplicity:

$$
\text { while }(i>0)\{i--;\} \quad \text { while }(1 \text { != null })\{1=1 . n e x t ;\}
$$

A useful size-relation for cost analysis is that the value of $i$ is always greater than 0 and decreases by 1 in each iteration, and that the longest path reachable from 1 is decreasing by 1 in each iteration.

Inferring size-relations is not straightforward: such relations might be the result of executing several statements, calling methods or loops. For instance, in our running example, the size relation for variable $i$ is the result of executing the method incr and is propagated through the loop in the procedure add. Fixpoint computation is often required. Fortunately, there are several abstract interpretation based approaches for inferring size-relations between integer variables [7], as well as between reference variables (in terms of longest path length) [20]. 


\subsection{The Notion of Size Relation}

In order to set up cost relations, we need, for each rule in the recursive representation, the calls-to size-relations between the variables in the head of the rule and the variables used in the calls (to rules) which occur in the body. Note that, given a rule $\mathrm{p}(\overline{\mathrm{x}}) \leftarrow \mathrm{G}, \overline{\mathrm{B}}_{\mathrm{k}},\left(\mathrm{q}_{1} ; \ldots ; \mathrm{q}_{\mathrm{n}}\right)$, each $\mathrm{b}_{\mathrm{i}} \in \overline{\mathrm{B}}_{\mathrm{k}}$ is either a bytecode or a call to another rule (which stems from the translation of a method invocation). We denote by calls $\left(\bar{B}_{k}\right)$ the set of all $b_{i}$ corresponding to a method call, and by bytecode $\left(\overline{\mathrm{B}}_{\mathrm{k}}\right)$ the set of all $\mathrm{b}_{\mathrm{i}}$ corresponding to other bytecodes.

Definition 1 (calls-to size-relations). Let $\mathcal{R}_{m}$ be the recursive representation of a method $m$, where each rule takes the form $\mathrm{p}(\overline{\mathrm{x}}) \leftarrow \mathrm{G}, \overline{\mathrm{B}}_{\mathrm{k}},\left(\mathrm{q}_{1}(\overline{\mathrm{y}}) ; \cdots ; \mathrm{q}_{\mathrm{n}}(\overline{\mathrm{y}})\right)$. The calls-to size-relations of $\mathcal{R}_{m}$ are triples of the form

$$
\left\langle\mathrm{p}(\overline{\mathrm{x}}), \mathrm{p}^{\prime}(\overline{\mathrm{z}}), \varphi\right\rangle \quad \text { where } \quad \mathrm{p}^{\prime}(\overline{\mathrm{z}}) \in \operatorname{calls}\left(\overline{\mathrm{B}}_{\mathrm{k}}\right) \cup\left\{\mathrm{p} \_\operatorname{cont}(\overline{\mathrm{y}})\right\}
$$

describing, for all rules, the size-relation between $\overline{\mathrm{x}}$ and $\overline{\mathbf{z}}$ when $\mathrm{p}^{\prime}(\overline{\mathbf{z}})$ is called, where $\mathrm{p}_{-} \mathrm{cont}(\overline{\mathrm{y}})$ refers to the program point immediately after $\overline{\mathrm{B}}_{\mathrm{k}}$. The sizerelation $\varphi$ is given as a conjunction of linear constraints $\mathrm{a}_{0}+\mathrm{a}_{1} \mathrm{v}_{1}+\cdots+\mathrm{a}_{\mathrm{n}} \mathrm{v}_{\mathrm{n}}$ op 0 , where op $\in\{=, \leq,<\}$, each $\mathrm{a}_{\mathrm{i}}$ is a constant and $\mathrm{v}_{\mathrm{k}} \in \overline{\mathrm{x}} \cup \overline{\mathrm{z}}$ for each $\mathrm{k}$.

Note that in the definition above there is no need to have separate relations for each $\mathrm{q}_{i}(\overline{\mathrm{y}})$ as, in the absence of exceptions, size relations are exactly the same for all of them, since they correspond to the same program point.

\subsection{Inferring Size Relations}

A simple, yet quite precise and efficient, size-relation analysis for the recursive representation of methods can be done in two steps: 1) compiling the bytecodes into the linear constraints they impose on variables; and 2) computing a bottomup fixpoint on the compiled rules using standard bottom-up fixpoint algorithms. Compilation into linear constraints is done by an abstraction function $\alpha_{\text {size }}$ which basically replaces guards and bytecodes by the constraints they impose on the corresponding variables. In general, each bytecode performing (linear) arithmetic operations is replaced by a corresponding linear constraint, and each bytecode which manipulates objects is compiled to linear constraints on the length of the longest reachable path from the corresponding variable [20]. Here are some examples of abstracting guards and bytecodes into linear constraints:

\begin{tabular}{|l|l|}
\hline$\alpha_{\text {size }}\left(\operatorname{iload}\left(l_{1}, \mathbf{s}_{0}\right)\right):=\left(l_{1}=\mathbf{s}_{0}\right)$ & $\frac{\alpha_{\text {size }}\left(\operatorname{guard}\left(\operatorname{icmpgt}\left(\mathbf{s}_{1}, \mathbf{s}_{0}\right)\right)\right):=\left(\mathbf{s}_{1}>\mathbf{s}_{0}\right)}{\alpha_{\text {size }}\left(\operatorname{iadd}\left(\mathbf{s}_{1}, \mathbf{s}_{0}, \mathbf{s}_{0}^{\prime}\right)\right):=\left(\mathbf{s}_{0}^{\prime}=\mathbf{s}_{0}+\mathbf{s}_{1}\right)}$ \\
\hline$\alpha_{\text {size }}\left(\operatorname{getfield}\left(\mathbf{s}_{1}, \mathbf{f}, \mathbf{s}_{1}^{\prime}\right)\right):=\left(\mathbf{s}_{1}^{\prime}<\mathbf{s}_{1}\right)$ \\
\hline
\end{tabular}

It is important to note that $\alpha_{\text {size }}$ uses the same name for the original variables in order to refer to their sizes. Compiling the rules of Ex. 3 results in:

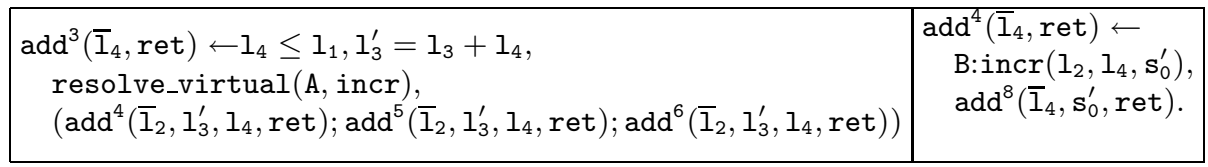


Example 5. Compiling all the rules corresponding to the program in Fig. 11 and computing a bottom-up fixpoint over an appropriate abstract domain [7] would result in the following calls-to size-relations for rules from Ex. 2,

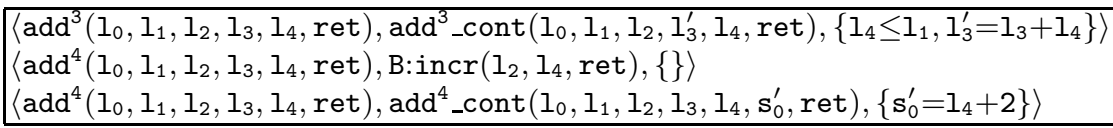

\section{Cost Relations for Java Bytecode}

We now present our approach to the automatic generation of cost relations which define the computational cost of the execution of a bytecode method. They are generated from the recursive representation of the method (Sec. 4) and by using the information inferred by the size analysis (Sec. 5). An important issue in order to obtain optimal cost relations is to find out the arguments which can be safely ignored in cost relations.

\subsection{Restricting Cost Relations to (Subsets of) Input Arguments}

Let us consider Block $k_{i d}$ in a CFG, represented by the rule c:m ${ }^{i d}\left(\overline{\mathrm{I}_{\mathrm{k}}}\right.$, ret $) \leftarrow \mathrm{G}, \overline{\mathrm{B}}_{\mathrm{h}}$, $\left(\mathrm{call}_{\mathrm{id}_{1}} ; \ldots ; \mathrm{call}_{\mathrm{id}_{\mathrm{j}}}\right)$ in which local and stack variables are no longer distinguishable. The cost function for $B l o c k_{i d}$ takes the form $C_{i d}:(\mathbb{Z})^{n} \rightarrow \mathbb{N}_{\infty}$, with $n \leq k$ argument positions, and where $\mathbb{Z}$ is the set of integers and $\mathbb{N}_{\infty}$ is the set of natural numbers augmented with a special symbol $\infty$, denoting unbounded.

Our aim here is to minimize the number $n$ of arguments which need to be taken into account in cost functions. As usual in cost analysis, we consider that the output argument ret cannot influence the cost of any block, so that it can be ignored in cost functions. Furthermore, it is sometimes possible to disregard some input arguments. For instance, in our running example, $I_{3}$ is an accumulating parameter whose value does not affect the control flow nor the cost of the program: it merely keeps the value of the temporary result.

Given a rule, the arguments which can have an impact on the cost of the program are those which may affect directly or indirectly the program guards (i.e., they can affect the control flow of the program), or are used as input arguments to external methods whose cost, in turn, may depend on the input size. Computing a safe approximation of the set of variables affecting a series of statements is a well studied problem in static analysis. To do this, we need to follow data dependencies against the control flow, and this involves computing a fixpoint. Our problem is slightly simpler than program slicing [21], since we do not need to delete redundant program statements; instead, we only need to detect relevant arguments. Given a rule $p(\overline{\mathrm{x}}) \leftarrow \operatorname{bod} y$ ( $p$ for short), $\hat{\mathrm{I}}_{p} \subseteq \overline{\mathrm{x}}$ is the sub-sequence of relevant variables for $p$. The sequence $\hat{1}_{P}$, obtained by union of sequences $\left\{\hat{\mathfrak{I}}_{p}\right\}_{p \in P}$ for a set $P$ of rules, keeps the ordering on variables.

Example 6. Given $p_{i}$, corresponding to Block $k_{i}$ in the graph of the running example, we are interested in computing which variables in this rule are relevant to program guards or external methods. For example, 1) when the execution flow 
reaches $p_{2}$, we execute the unconditional bytecode instructions in $p_{2}$ and move to the final block. As a result, there are no relevant variables for $p_{2}$, since none can have any impact on its cost, and $p_{2}$ does not reach any guards nor methods. 2) On the other hand, $p_{3}$ can reach the guards in $p_{4}, p_{5}$ and $p_{6}$, which take the form instanceof(-) and involve $l_{2}$. Also, the guard in $p_{3}$ itself, involving $l_{1}$ and $1_{4}$, can be recursively reached via the loop. Moreover, the call to the external method incr involves $l_{2}$ and $l_{4}$. After computing a fixpoint, we conclude that $\left.\hat{1}_{p_{3}}=\left\{I_{1}, l_{2}, l_{4}\right\} .3\right)$ We have $\hat{1}_{p_{8}}=\left\{l_{1}, l_{2}, s_{0}\right\}$; here, $s_{0}$ is also relevant since it affects $l_{4}$ (which in turn is involved in the guard of $p_{3}$, reachable from $p_{8}$ ).

\subsection{The Cost Relation}

Herein, we define the cost function $C_{i d}:(\mathbb{Z})^{n} \rightarrow \mathbb{N}_{\infty}$ for a $B l o c k_{i d}$ by means of a cost relation which consists of a set of cost equations. It will allow us to reason about the computational cost of the execution of the block $i d$. The intuitive idea is that, given the rule $\mathrm{p}(\overline{\mathrm{x}}) \leftarrow \mathrm{G}, \mathrm{B},\left(\mathrm{q}_{1} ; \ldots ; \mathrm{q}_{\mathrm{n}}\right)$ associated to $B l o c k_{i d}$, we generate:

- one cost equation which defines the cost of $\mathrm{p}$ as the cost of the statements in $\mathrm{B}$, plus the cost of its continuation, denoted p_cont;

- another cost equation which defines the cost of p_cont as either the cost of $\mathrm{q}_{1}$ (if its guard is satisfied), .., or the cost of $\mathrm{q}_{\mathrm{n}}$ (if its guard is satisfied).

We specify the cost of the continuation in a separate equation because the conditions for determining the alternative path $\mathrm{q}_{i}$ that the execution will take (with $i=1, \ldots, n)$ are only known at the end of the execution of $\mathrm{B}$; thus, they cannot be evaluated before $B$ is executed. In the definition below, we use the function $\alpha_{\text {guard }}$ to replace those guards which indicate the type of an object by the appropriate test (e.g., $\alpha_{\text {guard }}\left(\right.$ guard $\left(\right.$ instanceof $\left.\left.\left.\left(\mathrm{s}_{0},\{\mathrm{~B}\}\right)\right)\right):=\mathrm{s}_{0} \in \mathrm{B}\right)$. For guards on size relations, it is equivalent to $\alpha_{\text {size }}$.

Definition 2 (cost relation). Let $\mathcal{R}_{m}$ be the recursive representation of a method $m$ where each block takes the form $\mathrm{p}(\overline{\mathrm{x}}) \leftarrow \mathrm{G}_{\mathrm{p}}, \mathrm{B},\left(\mathrm{q}_{1}(\overline{\mathrm{y}}) ; \cdots ; \mathrm{q}_{\mathrm{n}}(\overline{\mathrm{y}})\right)$ and $\hat{1}_{\mathrm{p}}$ be its sequence of relevant variables. Let $\varphi$ be the calls-to size relation for $\mathcal{R}_{m}$ where each size relation is of the form $\left\langle\mathrm{p}(\overline{\mathrm{x}}), \mathrm{p}^{\prime}(\overline{\mathbf{z}}), \varphi_{\mathrm{p}^{\prime}(\overline{\mathbf{z}})}^{\mathrm{p}(\overline{\bar{x}})}\right\rangle$ for all $\mathrm{p}^{\prime}(\overline{\mathbf{z}}) \in \operatorname{call} \mathrm{s}(\mathrm{B})$ $\cup\{\mathrm{q}(\overline{\mathrm{y}})\}$ such that $\mathrm{q}(\overline{\mathrm{y}})$ refers to the program point immediately after $\mathrm{B}$. Then, we generate the cost equations for each block of the above form in $\mathcal{R}_{m}$ as follows:

$$
\begin{aligned}
& C_{p}\left(\hat{\mathrm{I}}_{p}\right)=\sum_{\text {bebytecode }(B)} T_{b}+\sum_{r(\bar{z}) \in \text { calls }(B)} C_{r}\left(\hat{\mathrm{I}}_{r}\right)+C_{p_{-} \text {cont }}\left(\cup_{i=1}^{n} \hat{\mathrm{I}}_{q_{i}}\right) \\
& C_{p_{-} \text {cont }}\left(\cup_{i=1}^{n} \hat{\mathrm{I}}_{q_{i}}\right)=\left\{\begin{array}{c}
C_{q_{1}}\left(\hat{\mathrm{I}}_{q_{1}}\right) \\
\ldots \\
C_{q_{n}}\left(\hat{\mathrm{I}}_{q_{n}}\right)
\end{array}\right. \\
& \bigwedge\left(\varphi_{r(\bar{z})}^{p(\bar{x})}\right) \wedge \varphi_{q(\bar{y})}^{p(\bar{x})} \\
& r(\bar{z}) \in \operatorname{calls}(B) \\
& \alpha_{\text {guard }}\left(G_{q_{1}}\right) \\
& \alpha_{\text {guard }}\left(G_{q_{n}}\right)
\end{aligned}
$$

where $T_{b}$ is the cost unit associated to the bytecode $b$. The cost relation associated to $\mathcal{R}_{m}$ and $\varphi$ is defined as the set of cost equations of its blocks.

Let us notice four points about the above definition. 1) The size relationships between the input variables provided by the size analysis are attached to the 
cost equation for $p$ (in Sect. 7 we discuss how to apply them). 2) Guards do not affect the cost: they are simply used to define the applicability conditions of the equations. 3) Arguments of the cost equations are only the relevant arguments to the block. In the equation for the continuation, we need to include the union of all relevant arguments to each of the subsequent blocks $q_{i}$.

The cost $T_{b}$ of an instruction $b$ depends on the chosen cost model. If our interest is merely on finding out the complexity or on approximating the number of bytecode statements which will be executed, then $T_{b}$ can be the same for all instructions. On the other hand, we may use more refined cost models in order to estimate the execution time of methods. Such models may assign different costs to different instructions. One approach might be based on the use of a profiling tool which estimates the value of each $T_{b}$ on a particular platform. (see, e.g., an application 14 for Prolog). It should be noted that, since we are not dealing with the problem of choosing a realistic cost model, a direct comparison between the result of our analysis and the actual measured run time (e.g., in milliseconds) cannot be done; instead, in this paper we focus only on the number of instructions to be executed.

Example 7. Consider the recursive representation in Ex. 2 (without irrelevant variables, as explained in Ex. 6). Consider the size relations derived in Ex. 5. by applying Def. 2] we obtain the following cost relations:

$$
\begin{aligned}
& C_{\text {add }}\left(1_{1}, 1_{2}\right)=C_{\text {add }}\left(1_{1}, 1_{2}\right) \\
& C_{\text {add }^{0}}\left(l_{1}, l_{2}\right) \quad=\mathrm{T}_{0}+C_{\text {add }^{1}}\left(l_{1}, l_{2}, l_{4}^{\prime}\right) \quad l_{4}^{\prime}=0 \\
& C_{\text {add } 1}\left(1_{1}, l_{2}, l_{4}\right)=\mathrm{T}_{1}+C_{\text {add }^{1} \text { _cont }}\left(1_{1}, 1_{2}, l_{4}\right)
\end{aligned}
$$

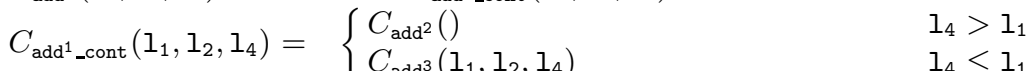

$$
\begin{aligned}
& C_{\text {add }^{2}}() \quad=\mathrm{T}_{2} \\
& C_{\text {add }^{3}}\left(l_{1}, l_{2}, l_{4}\right)=\mathrm{T}_{3}+C_{\text {add }^{3} \text { _cont }}\left(l_{1}, l_{2}, l_{4}\right)
\end{aligned}
$$

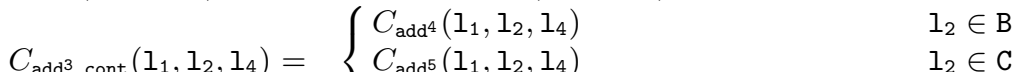

$$
\begin{aligned}
& C_{\text {add }^{3} \text { _cont }}\left(1_{1}, 1_{2}, 1_{4}\right)= \begin{cases}C_{\text {add }^{5}}\left(l_{1}, 1_{2}, 1_{4}\right) & 1_{2} \in \mathrm{C} \\
C_{\text {add }^{6}}\left(l_{1}, l_{2}, l_{4}\right) & 1_{2} \in \mathrm{A}\end{cases} \\
& C_{\text {add }^{4}}\left(l_{1}, l_{2}, l_{4}\right)=\mathrm{T}_{4}+C_{\mathrm{B}: \text { incr }}\left(\mathrm{l}_{2}, \mathrm{l}_{4}\right)+C_{\mathrm{add}^{8}}\left(\mathrm{l}_{1}, \mathrm{l}_{2}, \mathrm{~s}_{0}\right) \quad \mathrm{s}_{0}=\mathrm{l}_{4}+2 \\
& C_{\text {add }^{5}}\left(1_{1}, l_{2}, l_{4}\right)=\mathrm{T}_{5}+C_{\mathrm{C}: \text { incr }}\left(\mathrm{l}_{2}, \mathrm{l}_{4}\right)+C_{\mathrm{add}^{8}}\left(\mathrm{l}_{1}, \mathrm{l}_{2}, \mathrm{~s}_{0}\right) \quad \mathrm{s}_{0}=\mathrm{l}_{4}+3 \\
& C_{\text {add }^{6}}\left(l_{1}, l_{2}, l_{4}\right)=\mathrm{T}_{6}+C_{\mathrm{A}: \mathrm{incr}}\left(\mathrm{l}_{2}, \mathrm{l}_{4}\right)+C_{\mathrm{add}^{8}}\left(\mathrm{l}_{1}, \mathrm{l}_{2}, \mathrm{~s}_{0}\right) \quad \mathbf{s}_{0}=\mathrm{l}_{4}+1 \\
& C_{\text {add }^{8}}\left(\mathrm{l}_{1}, \mathrm{l}_{2}, \mathrm{~s}_{0}\right)=\mathrm{T}_{8}+C_{\mathrm{add}^{1}}\left(\mathrm{l}_{1}, \mathrm{l}_{2}, \mathrm{~s}_{0}\right)
\end{aligned}
$$

$\mathrm{T}_{\mathrm{B}_{\mathrm{i}}}$ denotes the sum of the costs of all bytecode instructions contained in Block $k_{i}$. For brevity, as the blocks 0, 2, 4, 5, 6, and 8 have a single-branched continuation, we merge their two equations. Note that the cost relation for the external method incr does not include the third argument since it is an output argument.

Demonstrating the correctness of our approach to cost analysis requires: (1) Defining the meaning of cost in terms of the Java bytecode operational semantics; (2) Inheriting that definition to a corresponding (equivalent) operational a semantics of the recursive representation. (3) Demonstrating that the cost relations describe the cost as defined in step 2. The first two steps are straightforward as the CFG and the recursive representation describe the behavior of the original program, in particular at each branching point we have several possibilities from 
which only one will be executed. The correctness of the third step stems from the facts that the cost relations are obtained from the recursive representation by replacing each bytecode by its cost, and that the size analysis provides us with information that can be used to compute (or approximate) the number of times we visit in each program point during the execution.

\section{Solving and Approximating Cost Functions}

The cost relations we presented in Sect. 6] allow reasoning about the computational cost of methods, provided that size analysis was effective. However, such cost relations generally depend on the cost of other calls (i.e., they are often recursive). It is thus convenient to obtain a closed form solution for the function which corresponds to the cost of the method. This can be done in two steps. The first one involves eliminating existential variables, i.e., those which do not occur in the left hand side, thus obtaining recurrence equations. The second step involves using existing tools for solving recurrence equations and/or computing upper or lower bounds for them.

\subsection{Obtaining Recurrence Equations}

First, we consider size relations which only contain equalities. Given an existential variable $y$, a size relation $\varphi$ and a sequence of (input) variables $\bar{x}$, we denote by solve $(y, \varphi, \bar{x})$ the operation which returns an expression $e$, with $\operatorname{Vars}(e) \subseteq \bar{x}$, such that $\varphi=(y=e)$. The result can be possibly $y$ itself if no other $e$ is found. For instance, for $\varphi_{2}$ in Ex. 5, the operation solve $\left(s_{0}^{\prime}, \varphi_{2},\left\langle l_{1}, l_{2}, l_{4}\right\rangle\right)$ returns $l_{4}+2$. This allows replacing equation (1) by equation (2):

$$
\begin{aligned}
& C_{\mathrm{add}^{4}}\left(\mathrm{I}_{1}, \mathrm{I}_{2}, \mathrm{I}_{4}\right)=\mathrm{T}_{4}+C_{\mathrm{B}: \text { incr }}\left(\mathrm{I}_{2}, \mathrm{I}_{4}\right)+C_{\mathrm{add}^{8}}\left(\mathrm{l}_{1}, \mathrm{I}_{2}, \mathrm{~s}_{0}\right) \\
& C_{\mathrm{add}^{4}}\left(\mathrm{l}_{1}, \mathrm{l}_{2}, \mathrm{l}_{4}\right)=\mathrm{T}_{4}+C_{\mathrm{B}: \text { incr }}\left(\mathrm{l}_{2}, \mathrm{l}_{4}\right)+C_{\mathrm{add}^{8}}\left(\mathrm{l}_{1}, \mathrm{l}_{2}, \mathrm{l}_{4}+2\right)
\end{aligned}
$$

where $\mathbf{s}_{0}$ is replaced by its solved form $\mathbf{l}_{4}+2$. Similarly, we can obtain recurrence equations for the cost of blocks add ${ }^{5}, \operatorname{add}^{6}$, and add ${ }^{0}$. This way, all cost equations in Ex. [7 are converted into recurrence equations.

There exist more complicated situations, in which size analysis needs to approximate information and it is only able to provide intervals in which the values of a variable may range, rather than equalities. Given a variable $y$, a size relation $\varphi$ and a sequence of variables $\bar{x}$, the operation interval $(\varphi, y, \bar{x})$ returns:

- An interval $\left[e_{1}, e_{2}\right]$ with $\left(\operatorname{Vars}\left(e_{1}\right) \cup \operatorname{Vars}\left(e_{2}\right)\right) \subseteq \bar{x}$, s.t. $\varphi \models\left(e_{1} \leq y \leq e_{2}\right)$.

- Otherwise, the same variable $y$.

For instance, consider the cost relation $C_{p}(\bar{x})=\sum T_{b}+C_{q}(y) \quad \varphi$, where $\left[e_{1}, e_{2}\right]=$ interval $(\varphi, y, \bar{x})$. As $y$ can vary within an interval, we can only now estimate upper and lower bounds for $C_{p}(\bar{x})$. To do so, we have to cover all possible variations of $y$ (i.e., the situation in which the value of $y$ moves faster and the one in which it moves slower). For this purpose, we can generate the following relation:

$$
C_{p}(\bar{x})=\left\{\begin{array}{l}
\sum T_{b}+C_{q}\left(e_{1}\right) \\
\sum T_{b}+C_{q}\left(e_{2}\right)
\end{array}\right.
$$


and then maximize or minimize the cost relation, depending on whether we want to approximate the upper or lower bound, respectively, as we explain below. In a more complicated case, the cost of $q$ might depend on a sequence of variables $\bar{y}$ rather than a single $y$, and the size analysis might provide intervals (not only equalities) for several of them. This leads to a more complex formalization not included due to lack of space.

\subsection{Approximating Recurrence Equations}

Algorithms for approximating recurrence equations have been studied by a number of researchers (see, e.g., 22]) and there are several systems available (e.g., Mathematica, Maxima, Maple, Matlab, CASLog). As already mentioned, it is not always possible to find closed form solutions for a set of recurrence equations. However, it turns out that it is quite often possible to find a closed form which is not a solution to the set of equations, but is guaranteed to be an upper (or lower) bound of the cost function. In many cases, finding an upper (or lower) bound can be sufficient. In particular, in the cost relations presented in Sect. 6. it is interesting to compute upper or lower bounds in two situations:

- when we have alternative branches corresponding to the second cost equation in Def. 2 (which represent a dynamic dispatch or a conditional branching),

- when we have intervals (rather than equalities) for the size relations of some variables, as explained in Sect. 7.1.

For the estimation of upper and lower bounds in such cases, we provide a modified version of the second equation in Def. 2 (the first one remains identical):

$$
C_{\mathrm{p}}(\bar{x})=\left\{\begin{array}{lll}
C_{\mathrm{q}_{1}}(\bar{x}) & & G_{\mathrm{q}_{1}} \\
C_{\mathrm{q}_{\mathrm{n}}}(\bar{x}) & \cdots & G_{\mathrm{q}_{\mathrm{n}}}
\end{array} \quad C_{\mathrm{p}}^{u p}(\bar{x})=\max \left\{\begin{array}{lll}
C_{\mathrm{q}_{1}}^{u p}(\bar{x}) & & \\
C_{\mathrm{q}_{\mathrm{n}}}^{u p}(\bar{x}) & \cdots & G_{\mathrm{q}_{1}}
\end{array}\right.\right.
$$

$\begin{array}{ll}\text { (a) Cost recurrence equation } C_{\mathrm{p}} & \text { (b) Upper bound of recurrence equation }\end{array}$

Similarly, the lower bound $C_{\mathrm{p}}^{\text {low }}(\bar{x})$ of $C_{\mathrm{p}}(\bar{x})$ is defined as $C_{\mathrm{p}}^{\text {up }}(\bar{x})$ but computing mins rather than maxs.

Example 8. Consider the upper bound $C_{\text {add }}^{u p}\left(\mathrm{l}_{1}, \mathrm{l}_{2}\right)$, obtained from the cost relation $C_{\text {add }}\left(1_{1}, I_{2}\right)$ in Ex. 7. We only show the cost equations for $C_{\text {add }}{ }^{3}$ :

$$
\begin{aligned}
& C_{\text {add }^{3}}^{u p}\left(1_{1}, 1_{2}, 1_{4}\right)=\mathrm{T}_{3}+C_{\text {add }^{3} \text { _cont }}^{u p}\left(1_{1}, 1_{2}, 1_{4}\right) \\
& C_{\text {add }^{3} \text { _cont }}^{u p}\left(1_{1}, 1_{2}, 1_{4}\right)=\max \begin{cases}C_{\text {add }^{4}}^{u p}\left(1_{1}, 1_{2}, 1_{4}\right) & 1_{2} \in \mathrm{B} \\
C_{\text {add }^{5}}\left(1_{1}, 1_{2}, 1_{4}\right) & 1_{2} \in \mathrm{C} \\
C_{\text {add }^{u}}^{u p}\left(1_{1}, 1_{2}, 1_{4}\right) & 1_{2} \in \mathrm{A}\end{cases}
\end{aligned}
$$

In this case, we can easily find the following closed form solution by isolating each of the different branches in $C_{\text {add }^{3} \text {-cont }}^{u p}$.

(a) if $\mathrm{l}_{2} \in \mathrm{A} \quad C_{\text {add }}^{\text {up }}\left(\mathrm{l}_{1}, \mathrm{l}_{2}\right)=\left(\mathrm{l}_{1}+1\right)\left(\mathrm{T}_{1}+\mathrm{T}_{3}+\mathrm{T}_{4}+\mathrm{T}_{\mathrm{A}: \text { incr }}+\mathrm{T}_{8}\right)+\mathrm{T}_{0}+\mathrm{T}_{1}+\mathrm{T}_{2}$

(b) if $\mathrm{I}_{2} \in \mathrm{B} \quad C_{\text {add }}^{\text {up }}\left(\mathrm{l}_{1}, \mathrm{I}_{2}\right)=\left(\mathrm{l}_{1} / 2+1\right)\left(\mathrm{T}_{1}+\mathrm{T}_{3}+\mathrm{T}_{4}+\mathrm{T}_{\mathrm{B} \text { : incr }}+\mathrm{T}_{8}\right)+\mathrm{T}_{0}+\mathrm{T}_{1}+\mathrm{T}_{2}$

(c) if $\mathrm{I}_{2} \in \mathrm{C} \quad C_{\text {add }}^{u p}\left(\mathrm{I}_{1}, \mathrm{I}_{2}\right)=\left(\mathrm{I}_{1} / 3+1\right)\left(\mathrm{T}_{1}+\mathrm{T}_{3}+\mathrm{T}_{4}+\mathrm{T}_{\mathrm{C}: \text { incr }}+\mathrm{T}_{8}\right)+\mathrm{T}_{0}+\mathrm{T}_{1}+\mathrm{T}_{2}$ 
We use $\mathrm{T}_{\mathrm{A}: \text { incr }}$ to denote the constant cost A:incr. The upper bound is $\max (a, b, c)$ and the lower bound is $\min (a, b, c)$. In any case, the cost is linear with the size of $l_{1}$. If $\mathrm{T}_{\mathrm{A}: \text { incr }}=\mathrm{T}_{\mathrm{B}: \text { incr }}=\mathrm{T}_{\mathrm{C} \text { :incr }}$ then $a$ is the upper bound and $c$ the lower bound.

Unfortunately, it is rather difficult to syntactically characterize the class of programs whose cost relations can be expressed in a closed form.

\section{Conclusion}

We have presented an automatic approach to the cost analysis of Java bytecode, based on generating at compile-time cost relations for an input bytecode program. Such relations are functions of input data which are informative by themselves about the computational cost, provided an accurate size analysis is used to establish relationships between the input arguments. Essentially, the sources of inaccuracy in size analysis are: 1) guards depending (directly of indirectly) on values which are not handled in the abstraction, e.g., non-integer values, numeric fields or multidimensional arrays, cyclic data-structures; 2) loss of precision due to the abstraction of (non-linear) arithmetic instructions and domain operations like widening. In such cases, we can still set up cost relations; however, they might not be useful if the size relationships are not precise enough.

To the best of our knowledge, our work presents the first approach to the automatic cost analysis of Java bytecode. Related work in the context of Java bytecode includes the work in the MRG project [3], which can be considered complementary to ours. MRG focuses on building a proof-carrying code [15] architecture for ensuring that bytecode programs are free from run-time violations of resource bounds. Also, the resource which has been studied in more depth is heap consumption, since applications to be deployed on devices with a limited amount of memory, such as smartcards, must be rejected if they require more memory than that available. Another related work is [6], where a resource usage analysis is presented. Again, this work focuses on memory consumption and it aims at verifying that the program executes in bounded memory by making sure that the program does not create new objects inside loops. The analysis has been certified by proving its correctness using the Coq proof assistant.

Acknowledgments. This work was funded in part by the Information Society Technologies program of the European Commission, Future and Emerging Technologies under the IST-15905 MOBIUS project, by the Spanish Ministry of Education (MEC) under the TIN-2005-09207 MERIT project, and the Madrid Regional Government under the S-0505/TIC/0407 PROMESAS project. S. Genaim was supported by a Juan de la Cierva Fellowship awarded by MEC.

\section{References}

1. A. V. Aho, R. Sethi, and J. D. Ullman. Compilers - Principles, Techniques and Tools. Addison-Wesley, 1986.

2. E. Albert, G. Puebla, and M. Hermenegildo. Abstraction-Carrying Code. In Proc. of LPAR'04, number 3452 in LNAI, pages 380-397. Springer-Verlag, 2005. 
3. D. Aspinall, S. Gilmore, M. Hofmann, D. Sannella, and I. Stark. Mobile Resource Guarantees for Smart Devices. In $C A S S I S^{\prime} 04$, number 3362 in LNCS. Springer, 2005.

4. G. Barthe, L. Beringer, P. Crégut, B. Grégoire, M. Hofmann, P. Müller, E. Poll, G. Puebla, I. Stark, and E. Vétillard. Mobius: Mobility, ubiquity, security: Objectives and progress report. In Trustworthy Global Computing'06, LNCS, 2007.

5. R. Benzinger. Automated higher-order complexity analysis. Theor. Comput. Sci., 318(1-2), 2004.

6. D. Cachera, D. Pichardie T. Jensen, and G. Schneider. Certified memory usage analysis. In FM'05, number 3582 in LNCS. Springer, 2005.

7. P. Cousot and N. Halbwachs. Automatic discovery of linear restraints among variables of a program. In Proc. POPL. ACM, 1978.

8. K. Crary and S. Weirich. Resource bound certification. In POPL. ACM, 2000.

9. R. Cytron, J. Ferrante, B. K. Rosen, M. N. Wegman, and F. K. Zadeck. Efficiently computing static single assignment form and the control dependence graph. TOPLAS, 13(4), 1991.

10. S. K. Debray and N. W. Lin. Cost analysis of logic programs. TOPLAS, 15(5), 1993.

11. S. K. Debray, P. López-García, M. Hermenegildo, and N.-W. Lin. Lower Bound Cost Estimation for Logic Programs. In Proc. ILPS'97. MIT Press, 1997.

12. G. Gomez and Y. A. Liu. Automatic time-bound analysis for a higher-order language. In Proc. of PEPM. ACM Press, 2002.

13. T. Lindholm and F. Yellin. The Java Virtual Machine Specification. AddisonWesley, 1996.

14. E. Mera, P. López-García, G. Puebla, M. Carro, and M. Hermenegildo. Combining Static Analysis and Profiling for Estimating Execution Times. In PADL'0\%, LNCS. Springer-Verlag, 2007. To appear.

15. G. Necula. Proof-Carrying Code. In POPL'97. ACM Press, 1997.

16. F. A. Rabhi and G. A. Manson. Using Complexity Functions to Control Parallelism in Functional Programs. TR. CS-90-1, Dept. of C.S., Univ. of Sheffield, UK, 1990.

17. M. Rosendhal. Automatic Complexity Analysis. In Proc. FPCA. ACM, 1989.

18. D. Sands. A naïve time analysis and its theory of cost equivalence. J. Log. Comput., 5(4), 1995.

19. F. Spoto. Julia: A Generic Static Analyser for the Java Bytecode. In Proc. of the 'th Workshop on Formal Techniques for Java-like Programs, FTfJP'2005, Glasgow, Scotland, July 2005. Available at www.sci.univr.it/ spoto/papers.html.

20. F. Spoto, P. M. Hill, and E. Payet. Path-length analysis for object-oriented programs. In Proc. EAAI, 2006.

21. F. Tip. A Survey of Program Slicing Techniques. J. of Prog. Lang., 3, 1995.

22. H. S. Wilf. Algorithms and Complexity. A.K. Peters Ltd, 2002.

23. R. Wilhelm. Timing analysis and timing predictability. In Proc. FMCO, LNCS. Springer-Verlag, 2004. 